\title{
ANALYSIS OF AN ANTIMICROBIAL RESISTANCE TRANSMISSION MODEL
}

\author{
JOHN SANGYEOB KIM $\dagger$ \\ ADVISER: ADOLFO J. RUMBOS $\ddagger$ \\ DEPARTMENT OF MATHEMATICS, POMONA COLLEGE
}

\begin{abstract}
We present an analysis of a system of differential equations that models the transmission dynamics of pathogens with antimicrobial resistance (AMR) in an intensive care unit (ICU) studied by Austin and Anderson (1999). In Austin and Anderson's four-dimensional compartmental model, patients and health care workers are viewed as hosts and vectors of the pathogens, respectively, and subdivided into uncolonized and colonized populations. In the analysis, we reduce the model to a two-dimensional non-autonomous system. Noting that the reduced system has an autonomous limiting system, we then apply the theory of asymptotically autonomous differential equations systems in the plane developed by Markus (1956) and extended by Thieme (1992, 1994), and later by Castillo-Chavez and Thieme (1995).

We first present a stability analysis of the limiting system and prove the existence of a locally asymptotically stable equilibrium point under a set of constraints expressed in terms of reproductive numbers. We then proceed to an asymptotic analysis of the non-autonomous, two-dimensional system by applying a Poincaré-Bendixson type trichotomy result proved by Thieme $(1992,1994)$. In particular, we establish that any forward bounded trajectory of the non-autonomous system that starts within a defined rectangular region will converge toward the equilibrium point of the limiting system, provided that certain conditions given in terms of the reproductive numbers are satisfied.

KEYWORDS. antimicrobial resistance; transmission dynamics; compartmental model; non-autonomous system; limiting system; locally asymptotically stable equilibrium
\end{abstract}

\section{INTRODUCTION}

Antimicrobial resistance (AMR) is the ability of bacteria to resist the effects of antibiotics. When a high density patient population is exposed to heavy antibiotic use and frequent contact with healthcare staff, the risk of cross infection increases and intensive care units (ICUs) in hospitals become a hotbed of antibiotic resistant bacteria [Str98]. In light of such medical practices, we examine transmission dynamics of antibiotic resistant pathogens in hospitals.

This research was supported by the Kenneth Cooke Summer Research Fellowship awarded to the author in the summer of 2017 at Pomona College, Claremont, California. The author would like to thank the editor, editorial associate, and anonymous referees for their careful reading of the manuscript and constructive feedback.

$\dagger$ e-mail: sababi0314@gmail.com.

$\ddagger e$-mail: arumbos@pomona.edu. 
The system of differential equations

$$
\left\{\begin{aligned}
\frac{d X_{p}}{d t} & =(1-\phi) \Lambda-\mu X_{p}-c b_{p} Y_{h} X_{p} \\
\frac{d Y_{p}}{d t} & =\phi \Lambda-\mu Y_{p}+c b_{p} Y_{h} X_{p} \\
\frac{d X_{h}}{d t} & =-c b_{h} Y_{p} X_{h}+\gamma Y_{h} \\
\frac{d Y_{h}}{d t} & =c b_{h} Y_{p} X_{h}-\gamma Y_{h}
\end{aligned}\right.
$$

models the transmission dynamics of pathogens with AMR in a hospital unit. The model (1) was studied by Austin and Anderson [AA99]. Figure 1, which is similar to the one used in [AA99], shows the flow diagram associated with the system (1). The population in the hospital unit is divided into two groups: patients,

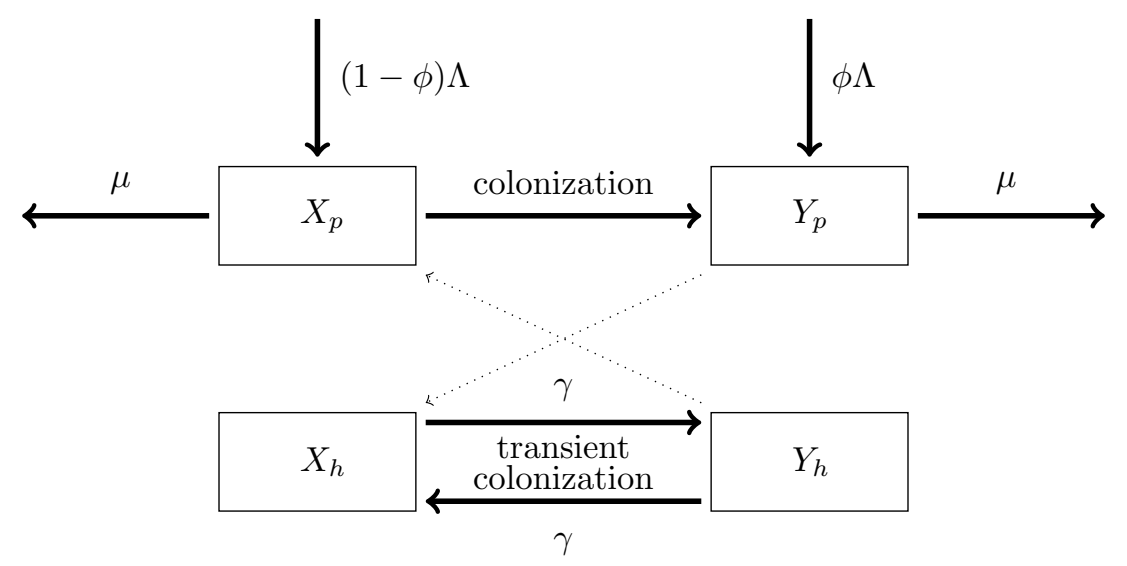

Figure 1. Flow Diagram for the Austin-Anderson Model.

identified with the subscript $p$, and health care workers (HCWs), identified with the subscript $h .^{1}$ In turn, patients and HCWs are subdivided into two groups each: uncolonized, denoted by the variable $X$, and colonized, denoted by the variable $Y$. Colonized individuals are those who have microorganisms in their bodies (e.g., bacteria) that are resistant to antimicrobial medications (e.g., antibiotics). Thus, $Y_{p}(t)$ denotes the number of patients in the unit that carry AMR microorganism at time $t$, and $Y_{h}(t)$ denotes the number of HCWs that have been colonized by the AMR microorganisms at time $t$. By the same token, $X_{p}(t)$ denotes the number of patients that do not have the AMR microorganisms in their bodies, but are susceptible to being colonized by the microorganisms, at time $t$. Similarly, $X_{h}(t)$ denotes the number of uncolonized HCWs at time $t$. Austin and Anderson [AA99] distinguish between infection and colonization. An infection occurs when germs are

\footnotetext{
${ }^{1}$ While D'Agata et al. [EDW07] point out further complexities of the transmission dynamics, such as differentiating between types of HCW (nurses and physicians) and their interactions with patients, we stick to the original division for simplicity.
} 
in or on the body causing signs and symptoms. Colonization occurs when germs are in or on the body, but do not necessarily make the person sick.

In the system (1), $\Lambda$ is the patient admission rate, in units of population per time. A fraction, $\phi \Lambda$, is colonized ( $\phi$ is called the prevalence of colonization at admission); so that, a fraction $(1-\phi) \Lambda$ is uncolonized.

The parameter $\mu$ in the system (1) and the flow diagram in Figure 1 is the discharge rate; so that, $1 / \mu$ is a patient's average length of stay in the unit (typically measured in days).

It was assumed in [AA99] that transmission of the AMR microorganisms is achieved solely through contact; that is, the microorganisms are often passed from person to person by contaminated hands of HCWs, or colonization spreads directly to people after they touch surfaces that are contaminated with the AMR microorganisms. Accordingly, the parameter $c$ in the system (1) is the contact rate, measured in number of contacts per unit time, and $b_{p}$ denotes the probability of transmission from $\mathrm{HCW}$ to patient per contact, measured in units of $1 /$ (contact $\times$ population); that is, the probability that a patient is colonized by the AMR microorganisms in one contact with a $\mathrm{HCW}$ that is colonized. Thus, $c b_{p}$ is the rate of colonization because of contact with one HCW per unit of time. Similarly, $b_{h}$ is the probability of transmission from patient to HCW per contact; that is, $b_{h}$ is probability of colonization of a $\mathrm{HCW}$ in one contact with a colonized patient; consequently, $c b_{h}$ is the rate of colonization of HCWs by contact with one colonized patient.

Given the assumption about the transmission of AMR microorganisms stated above, the HCWs act as vectors of transmission. This is indicated in the flow diagram in Figure 1 by the dotted arrows. Thus, the ideas used in modeling vectorborne diseases can be applied in the modeling of AMR microorganisms transmission. This is precisely what Austin and Anderson did when they adapted a malaria model developed by Ross and MacDonald in 1911 and 1957, respectively, [MD13]. In this setting, patients are viewed as hosts. They are either uncolonized (susceptible to being colonized) or colonized by AMR microorganisms through contact with colonized HCWs.

As stated previously, HCWs are viewed as vectors. They are either uncolonized through effective hand-washing, or transiently colonized through interaction with other HCWs (transient colonization). The parameter $\gamma$ in the system (1) and in the flow diagram in Figure 1 is the transient colonization rate; accordingly, $1 / \gamma$ is the average duration of transient colonization (typically measured in hours); thus, $1 / \gamma$ represents the average time a colonized HCW remains colonized until he or she becomes uncolonized through means of hand-washing, for instance. ${ }^{2}$

Finally, Austin and Anderson [AA99] also assumed that, once patients become colonized by the AMR microorganisms, they remain colonized for the duration of their stay in the hospital unit.

\footnotetext{
${ }^{2}$ We are aware that modeling of nosocomial epidemics should take account of not only patientHCW hygiene measures, but also environmental infection transmission pathways, as Browne and Webb note in [BW15]. They show that more effective cleaning of rooms can substantially increase the probability of bacterial extinction; this is not incorporated into the system analyzed in this paper, where we only take into account one hygiene measure to be consistent with model (1).
} 
Adding the last two differential equations in the system (1), we obtain that

$$
\frac{d}{d t}\left[X_{h}+Y_{h}\right]=0, \quad \text { for all } t,
$$

from which we conclude that the total number of HCWs remains constant. We denote the total number of HCWs by $N_{h}$; so that,

$$
X_{h}(t)+Y_{h}(t)=N_{h}, \quad \text { for all } t .
$$

In view of equation (2), the four-dimensional system (1) can be reduced to the three-dimensional system

$$
\left\{\begin{aligned}
\frac{d X_{p}}{d t} & =(1-\phi) \Lambda-\mu X_{p}-c b_{p} Y_{h} X_{p} \\
\frac{d Y_{p}}{d t} & =\phi \Lambda-\mu Y_{p}+c b_{p} Y_{h} X_{p} \\
\frac{d Y_{h}}{d t} & =c b_{h} Y_{p}\left(N_{h}-Y_{h}\right)-\gamma Y_{h}
\end{aligned}\right.
$$

where $N_{h}$ is the constant number of HCWs. A further reduction in dimension can be attained by adding the first two differential equations of the system (3) to obtain

$$
\frac{d}{d t}\left[X_{p}+Y_{p}\right]=\Lambda-\mu\left(X_{p}+Y_{p}\right) ;
$$

so that, setting

$$
N_{p}(t)=X_{p}(t)+Y_{p}(t), \quad \text { for all } t,
$$

the total number of patients in the unit at time $t$, we have that

$$
\frac{d N_{p}}{d t}=\Lambda-\mu N_{p} .
$$

Assuming that the total number of patients at time $t_{0}$ is $N_{p_{0}}$, the solution of the differential equation (5) satisfying this initial condition is given by

$$
N_{p}(t)=\frac{\Lambda}{\mu}+\left(N_{p_{0}}-\frac{\Lambda}{\mu}\right) e^{-\mu\left(t-t_{0}\right)}, \quad \text { for all } t \geqslant t_{0} .
$$

Now, in view of equations (4) and (6), we see that knowing $X_{p}$ will allow us to compute $Y_{p}$ (in terms of $X_{p}$ and $N_{p}$ ). Consequently, the three-dimensional system (3) can be reduced to the two-dimensional system

$$
\left\{\begin{aligned}
\frac{d X_{p}}{d t} & =(1-\phi) \Lambda-\mu X_{p}-c b_{p} Y_{h} X_{p} \\
\frac{d Y_{h}}{d t} & =c b_{h}\left(N_{p}(t)-X_{p}\right)\left(N_{h}-Y_{h}\right)-\gamma Y_{h},
\end{aligned}\right.
$$

where $N_{p}(t)$ is given in equation (6).

Thus, we have succeeded in reducing the Austin-Anderson system (1) to the two-dimensional system (7). Note that, unlike the systems (1) and (3) which are autonomous, the two-dimensional system (7) is non-autonomous. Thus, the simplification brought on by the reduction of dimension is tempered by the fact 
that the reduced system (7) is non-autonomous, and therefore harder to analyze. Note, however, that by virtue of equation (6),

$$
\lim _{t \rightarrow \infty} N_{p}(t)=\frac{\Lambda}{\mu} ;
$$

so that, the non-autonomous system (7) has a limiting system

$$
\left\{\begin{aligned}
\frac{d X}{d t} & =(1-\phi) \Lambda-\mu X-c b_{p} Y X \\
\frac{d Y}{d t} & =c b_{h}\left(\frac{\Lambda}{\mu}-X\right)\left(N_{h}-Y\right)-\gamma Y,
\end{aligned}\right.
$$

as $t \rightarrow \infty$, in view of the limit fact in (8), which is an autonomous system in the variables $X$ and $Y$. We note that the variables $X$ and $Y$ are different from the variables $X_{p}$ and $Y_{p}$, respectively. We introduce new variables to distinguish the non-autonomous system (7) from its limiting system (9).

We will denote the limiting value of $N_{p}(t)$ in the asymptotic expression (8) by $\bar{N}_{p}$ so that as $t \rightarrow \infty$,

$$
\bar{N}_{p}=\frac{\Lambda}{\mu} .
$$

The stability analysis of the long-term behavior of the system (1) outlined in [AA99] is based on the analysis of a limiting system related to the system (9); in fact, the limiting system in [AA99] involves $Y_{p}$ and $Y_{h}$, and not $X_{p}$ and $Y_{h}$ as we decided to do here. It is not immediately obvious that the stability properties of the non-autonomous system (7) are determined by properties of the autonomous system (9). The goal of this paper is to provide a mathematical justification for the analysis of the AMR transmission system presented in [AA99]. This involves application of the theory of asymptotically autonomous differential equations (see for instance, [Mar56], [Thi92] and [Thi94]). This approach was used by CastilloChavez and Thieme [CCT95] in the analysis of some models in epidemiology that give rise to asymptotically autonomous two-dimensional systems.

In Section 2 we present a thorough analysis of the autonomous limiting system (9). In Section 3 we apply the theory of asymptotically autonomous differential equations systems in the plane developed by Markus in [Mar56] and extended by Thieme in [Thi92] and [Thi94], and later by Castillo-Chavez and Thieme in [CCT95], to obtain as much information about the long-term properties of the nonautonomous system (7). Finally, in Section 4, we return to the Austin-Anderson AMR transmission model discussed in this section.

\section{Stability Analysis of the Limiting System}

In this section we present a stability analysis of the two-dimensional, autonomous system (9). Before we proceed with the analysis, we introduce some dimensionless parameters that will be helpful in understanding the long-term behavior of colonization by AMR microorganisms in a hospital unit. These parameters are the reproductive numbers

$$
R_{h}=\frac{c b_{h} \bar{N}_{p}}{\gamma}
$$




$$
R_{p}=\frac{c b_{p} N_{h}}{\mu}
$$

and

$$
R_{0}=R_{h} R_{p}=\frac{m c^{2} b_{h} b_{p} \bar{N}_{p}^{2}}{\gamma \mu}
$$

where $\bar{N}_{p}$ is as given in equation (10), and

$$
m=\frac{N_{h}}{\bar{N}_{p}},
$$

is the staff-patient ratio.

As noted in [EDW07], to facilitate the analysis of the system, we can regroup the model parameters so that they can be expressed in terms of the reproductive numbers. The reproductive number $R_{h}$ in equation (11) is the number of secondary colonizations of patients resulting from contact with one colonized $\mathrm{HCW}$; the reproductive number $R_{p}$ in equation (12) is the number of uncolonized HCWs that get colonized because of contact with one colonized patient in a hospital unit. Since the transmission of AMR microorganisms goes from colonized patients to HCWs to patients, the overall reproductive number for transmission, $R_{0}$, is the product of $R_{h}$ and $R_{p}$, as displayed in equation (13).

Introducing the dimensionless variables

$$
x=\frac{X}{\bar{N}_{p}}, \quad y=\frac{Y}{N_{h}}, \quad \text { and } \quad \tau=\mu t,
$$

we can rewrite the system (9) in dimensionless form

$$
\left\{\begin{aligned}
\frac{d x}{d \tau} & =1-\phi-x-\alpha_{1} x y \\
\frac{d y}{d \tau} & =\alpha_{2}(1-x)(1-y)-\alpha_{3} y
\end{aligned}\right.
$$

where

$$
\begin{aligned}
& \alpha_{1}=\frac{c b_{p} N_{h}}{\mu}, \\
& \alpha_{2}=\frac{c b_{h} \bar{N}_{p}}{\mu},
\end{aligned}
$$

and

$$
\alpha_{3}=\frac{\gamma}{\mu}
$$

are dimensionless parameters. For future reference, we express the parameters $\alpha_{1}$, $\alpha_{2}$ and $\alpha_{3}$ in equations (16)-(18) in terms of the reproductive numbers $R_{h}$ and $R_{p}$ in equations (11) and (12), respectively:

$$
\alpha_{1}=R_{p},
$$

and

$$
\frac{\alpha_{2}}{\alpha_{3}}=R_{h} .
$$


We now proceed to analyze the system (15). Figure 2 shows a sketch of the nullclines of the limiting system (15). The $x$-nullcline is the graph of the equation

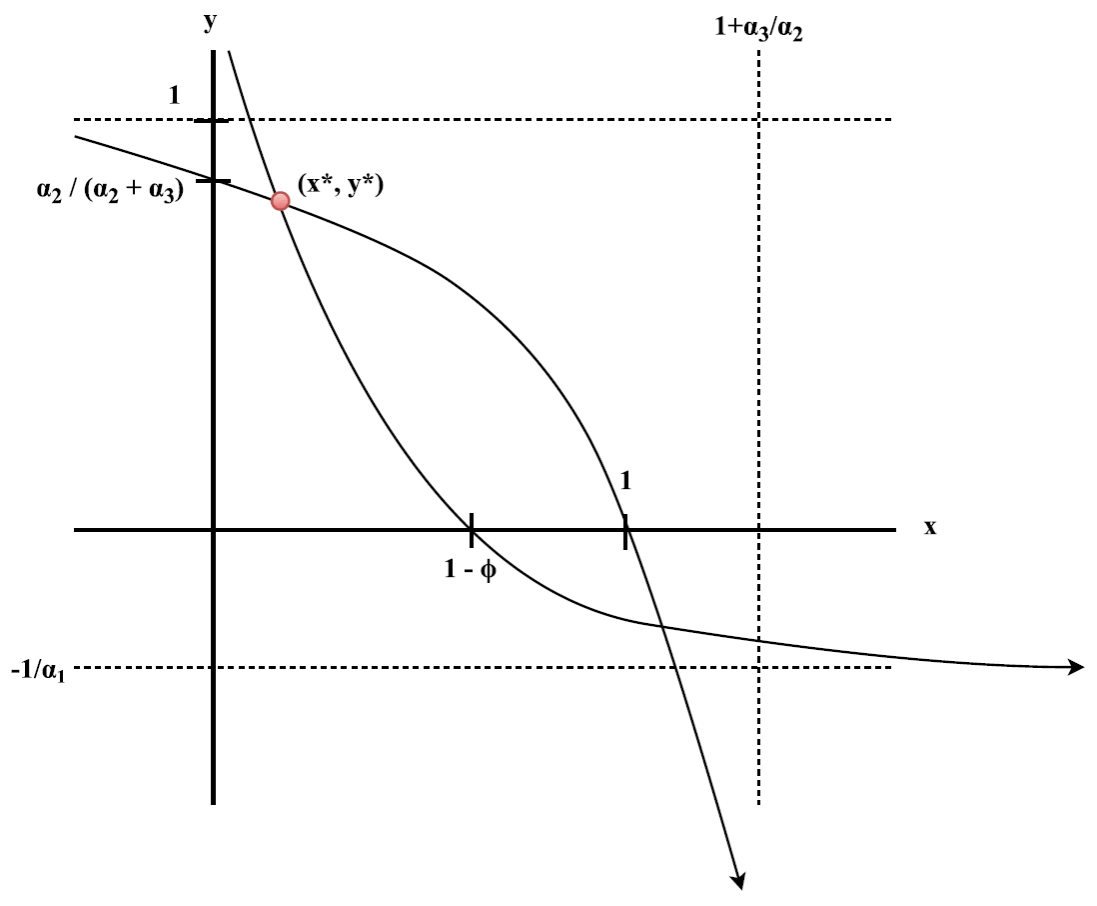

Figure 2. Nullclines of the limiting system (15).

$$
y=\frac{1-\phi}{\alpha_{1} x}-\frac{1}{\alpha_{1}},
$$

which has $1-\phi$ as its $x$-intercept, the $y$-axis as a vertical asymptote, and the line

$$
y=-\frac{1}{\alpha_{1}}
$$

as a horizontal asymptote (shown as a dotted line in Figure 2).

The $y$-nullcline is the graph of the equation

$$
y=\frac{\alpha_{2}(1-x)}{\alpha_{3}+\alpha_{2}(1-x)},
$$

which has the line $y=1$ as a horizontal asymptote and the line

$$
x=1+\frac{\alpha_{3}}{\alpha_{2}}
$$

as a vertical asymptote (these are shown as dotted lines in Figure 2). Note that the $y$-nullcline has $x$-intercept 1 and $y$-intercept $\frac{\alpha_{2}}{\alpha_{2}+\alpha_{3}}$.

The sketch in Figure 2 shows that the $x$-nullcline and the $y$-nullcline intersect at a point below the $x$-axis and at a point in the first quadrant that we denote by $\left(x^{*}, y^{*}\right)$. There are other branches of the nullclines that do not intersect and therefore are not shown in Figure 2. Thus, the limiting system (15) has two equilibrium 
points. We will focus our attention on the equilibrium point $\left(x^{*}, y^{*}\right)$ in the first quadrant. We first note that the coordinates of this equilibrium point satisfy the estimates

$$
0 \leqslant y^{*}<\frac{\alpha_{2}}{\alpha_{2}+\alpha_{3}}
$$

and

$$
0<x^{*} \leqslant 1-\phi .
$$

In view of the estimate for $x^{*}$ in inequality (22), we see that

$$
0<x^{*} \leqslant 1-\phi \leqslant 1,
$$

since the prevalence of colonization at admissions, $\phi$, is a proportion between 0 and 1.

We will next determine parameter conditions that will guarantee that the equilibrium point $\left(x^{*}, y^{*}\right)$ is (locally) asymptotically stable. To do this we apply the Principle of Linearized Stability (see, for instance, [Arn92, page 212]) to the vector field

$$
F(x, y)=\left(\begin{array}{c}
1-\phi-x-\alpha_{1} x y \\
\alpha_{2}(1-x)(1-y)-\alpha_{3} y
\end{array}\right), \quad \text { for }(x, y) \in \mathbb{R}^{2},
$$

at the equilibrium point $\left(x^{*}, y^{*}\right)$.

The derivative of the map $F: \mathbb{R}^{2} \rightarrow \mathbb{R}^{2}$ defined in equation (23) evaluated at $\left(x^{*}, y^{*}\right)$ is

$$
D F\left(x^{*}, y^{*}\right)=\left(\begin{array}{cc}
-1-\alpha_{1} y^{*} & -\alpha_{1} x^{*} \\
-\alpha_{2}\left(1-y^{*}\right) & -\alpha_{2}\left(1-x^{*}\right)-\alpha_{3}
\end{array}\right) .
$$

The trace of the Jacobian matrix in equation (24) is

$$
\tau\left(x^{*}, y^{*}\right)=-1-\alpha_{1} y^{*}-\alpha_{2}\left(1-x^{*}\right)-\alpha_{3}
$$

and the determinant is

$$
\Delta\left(x^{*}, y^{*}\right)=\left(1+\alpha_{1} y^{*}\right)\left[\alpha_{2}\left(1-x^{*}\right)+\alpha_{3}\right]-\alpha_{1} \alpha_{2} x^{*}\left(1-y^{*}\right) .
$$

From the the constraints on the coordinates of $\left(x^{*}, y^{*}\right)$ in inequalities (21) and (22), and the assumption that the parameters $\alpha_{1}, \alpha_{2}$, and $\alpha_{3}$ are positive, it follows from equation (25) that

$$
\tau\left(x^{*}, y^{*}\right)<0 .
$$

Next, we use the fact that

$$
0 \leqslant y^{*}<1
$$

according to inequality (21), to estimate

$$
\frac{\Delta\left(x^{*}, y^{*}\right)}{1-y^{*}}=\frac{1+\alpha_{1} y^{*}}{1-y^{*}}\left[\alpha_{2}\left(1-x^{*}\right)+\alpha_{3}\right]-\alpha_{1} \alpha_{2} x^{*} .
$$

Using the fact that

$$
\frac{1+\alpha_{1} y}{1-y} \geqslant 1, \quad \text { for } 0 \leqslant y<1
$$

we get

$$
\frac{\Delta\left(x^{*}, y^{*}\right)}{1-y^{*}} \geqslant\left[\alpha_{2}\left(1-x^{*}\right)+\alpha_{3}\right]-\alpha_{1} \alpha_{2} x^{*} .
$$

Lastly, in view of inequality (22),

$$
0<x^{*} \leqslant 1,
$$


and we use such estimate for $x^{*}$ and get

$$
\frac{\Delta\left(x^{*}, y^{*}\right)}{1-y^{*}} \geqslant \alpha_{2}+\alpha_{3}-\alpha_{2}\left(1+\alpha_{1}\right) x^{*} .
$$

Observe that

$$
y=\alpha_{2}+\alpha_{3}-\alpha_{2}\left(1+\alpha_{1}\right) x
$$

is the equation of a straight line with $y$-intercept $\alpha_{2}+\alpha_{3}$ and $x$-intercept

$$
\frac{\alpha_{2}+\alpha_{3}}{\alpha_{2}\left(1+\alpha_{1}\right)}=\frac{1+\frac{1}{R_{h}}}{1+R_{p}},
$$

where we have used the identities in (19) and (20). Consequently, it follows from inequality (28) that

$$
\frac{\Delta\left(x^{*}, y^{*}\right)}{1-y^{*}}>0, \quad \text { provided that } x^{*}<\frac{1+\frac{1}{R_{h}}}{1+R_{p}},
$$

from which we obtain that

$$
\Delta\left(x^{*}, y^{*}\right)>0, \quad \text { provided that } x^{*}<\frac{1+\frac{1}{R_{h}}}{1+R_{p}},
$$

in view of the estimate for $y^{*}$ in inequality (21). We consider two cases:

(i) $R_{0}<1$ or

(ii) $R_{0} \geqslant 1$,

where $R_{0}$ is the reproductive number defined in equation (13).

In case (i), using the definition of $R_{0}$ in inequality (13),

$$
R_{p} R_{h}<1,
$$

from which we get that

so that,

$$
\frac{1}{R_{h}}>R_{p}
$$

$$
\frac{1+\frac{1}{R_{h}}}{1+R_{p}}>1
$$

Now, recall that in view of the estimate for $x^{*}$ in inequality (22), we see that

$$
0<x^{*} \leqslant 1-\phi \leqslant 1 .
$$

Consequently, by virtue of inequality (30),

$$
0<x^{*}<\frac{1+\frac{1}{R_{h}}}{1+R_{p}},
$$

from which we see that the condition for the statement (29) to hold true is satisfied regardless of the value of $\phi$. Thus,

$$
\Delta\left(x^{*}, y^{*}\right)>0, \quad \text { provided that } R_{0}<1 .
$$

On the other hand, in case (ii) we have that

$$
R_{p} R_{h} \geqslant 1 \text {, }
$$


which implies that

$$
\frac{1}{R_{h}} \leqslant R_{p}
$$

so that,

$$
\frac{1+\frac{1}{R_{h}}}{1+R_{p}} \leqslant 1 .
$$

Thus, in case (ii), in view of the estimate for $x^{*}$ in inequality (22), we get from the statement (29) that

$$
\Delta\left(x^{*}, y^{*}\right)>0, \quad \text { provided that } R_{0} \geqslant 1 \text { and } 1-\phi<\frac{R_{h}+1}{R_{h}+R_{0}} .
$$

Combining statements (31) and (32) together, we conclude that the determinant of the linearization (24) of the limiting system (15) is positive in the following cases:

(i) $R_{0}<1$, and

(ii) $R_{0} \geqslant 1$ and $1-\phi<\frac{R_{h}+1}{R_{h}+R_{0}}$.

Since the trace of the matrix in equation (24) is negative, according to inequality (27), the real parts of the eigenvalues of the linearization of the limiting system (15) are negative in the cases (i) and (ii) above. Consequently, by the Principle of Linearized Stability, the equilibrium point $\left(x^{*}, y^{*}\right)$ is (locally) asymptotically stable, provided that the conditions (i) or (ii) hold true.

Phase-plane analysis of the limiting system (15) suggests that trajectories starting in the region determined by

$$
0 \leqslant x_{0} \leqslant 1 \text { and } y_{0} \geqslant 0
$$

will tend towards the asymptotically stable equilibrium point $\left(x^{*}, y^{*}\right)$ as $t \rightarrow \infty$ under the conditions (i) or (ii) stated above. Figure 3 shows a sketch of the phaseportrait of the system (15) obtained using the Java version of pplane ${ }^{3}$ with parameter values $\phi=0.7, \alpha_{1}=0.5, \alpha_{2}=2$ and $\alpha_{3}=0.5$.

The sketch in Figure 3 also suggests that the phase-portrait of the limiting system (15) has no cycles. This observation can be established using the BendixsonDulac Criterion (see, for instance, [Str00, p. 202]). Indeed, the divergence of the vector field $F$ in (23),

$$
\operatorname{div} F(x, y)=-1-\alpha_{1} y-\alpha_{2}(1-x)-\alpha_{3},
$$

is strictly negative for $0 \leqslant x \leqslant 1$ and $y \geqslant 0$, and for positive values of the parameters $\alpha_{1}, \alpha_{2}$, and $\alpha_{3}$. Indeed, it follows from equation (33) that

$$
\operatorname{div} F(x, y) \leqslant-1, \quad \text { for } 0 \leqslant x \leqslant 1 \text { and } y \geqslant 0 .
$$

Thus, using the continuity of $\operatorname{div} F$, we obtain from inequality (34) that there exists $\varepsilon>0$ such that

$$
\operatorname{div} F(x, y)<0, \quad \text { for }-\varepsilon<x<1+\varepsilon \text { and } y>-\varepsilon .
$$

This fact will be important in the analysis of the non-autonomous system (7) to be presented in the next section.

${ }^{3}$ pplane is copyrighted in the name of John C. Polking, Department of Mathematics, Rice University, https://math.rice.edu/ dfield/dfpp.html 


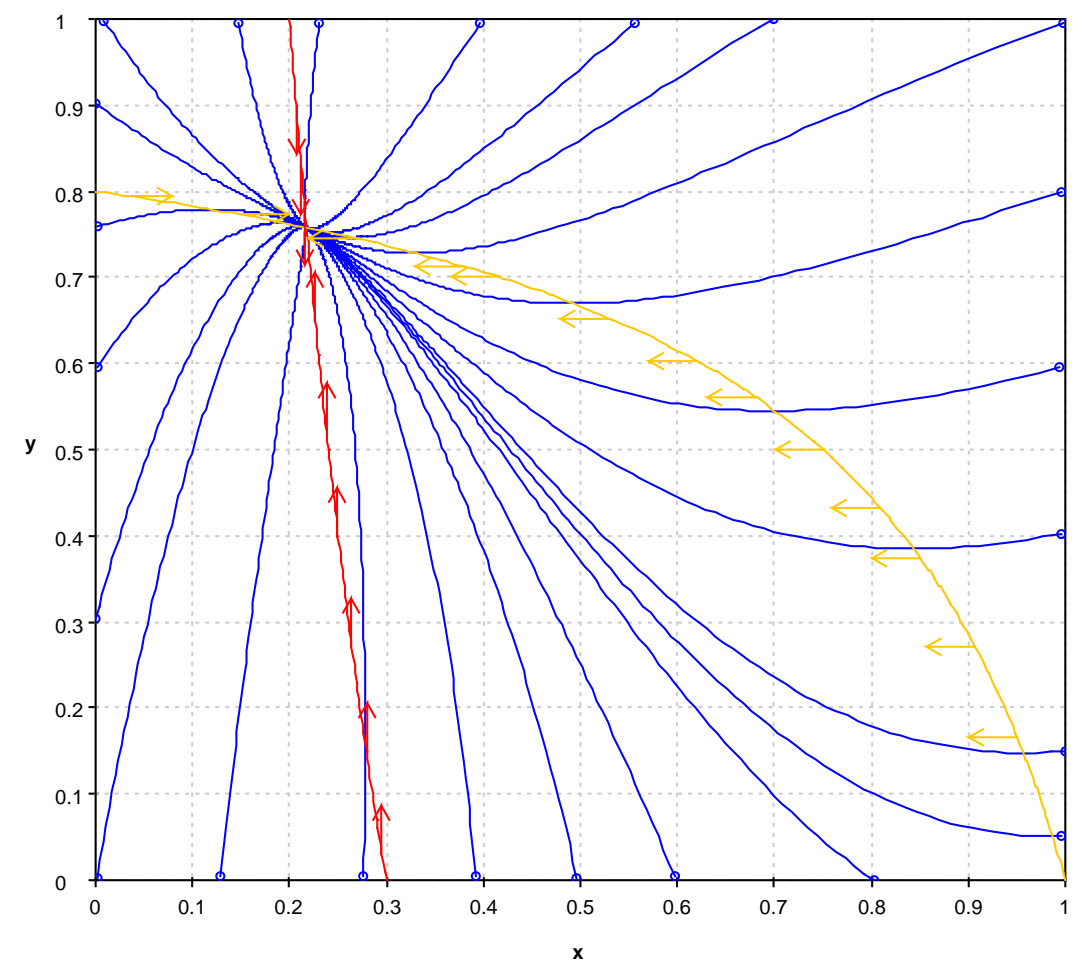

FiguRE 3. Sketch of phase-portrait of limiting system (15) with $\phi=0.7, \alpha_{1}=0.5, \alpha_{2}=2$ and $\alpha_{3}=0.5$. Note that trajectories starting in the region determined by $0 \leqslant x_{0} \leqslant 1$ and $y_{0} \geqslant 0$ will tend towards the asymptotically stable equilibrium point, which is a sink in the first quadrant.

We now complete the stability analysis of the limiting system (9). We have already analyzed its nondimensionalized version through system (15). We found that the nondimensionalized system (15) has an equilibrium point, $\left(x^{*}, y^{*}\right)$, in the first quadrant that is locally asymptotically stable, provided that either (i) $R_{0}<1$, or (ii) $R_{0} \geqslant 1$ and $1-\phi<\frac{R_{h}+1}{R_{h}+R_{0}}$, where $R_{0}$ and $R_{h}$ are the reproductive numbers defined in equations (11)-(13). Accordingly, in view of variables (14), the limiting system (9) has an equilibrium point $\left(X^{*}, Y^{*}\right)$ in the first quadrant where

$$
X^{*}=\frac{\Lambda}{\mu} x^{*} \quad \text { and } \quad Y^{*}=N_{h} y^{*} .
$$

By what we have shown in this section, the equilibrium point $\left(X^{*}, Y^{*}\right)$ is (locally) asymptotically stable, provided that either (i) $R_{0}<1$, or (ii) $R_{0} \geqslant 1$ and $1-\phi<$ $\frac{R_{h}+1}{R_{h}+R_{0}}$. We have also noted that, as a consequence of the Bendixson-Dulac Criterion, the nondimensionalized limiting system (15) has no cycles in the region determined by $-\varepsilon<x<1+\varepsilon$ and $y>-\varepsilon$, for some $\varepsilon>0$ (see inequality (35)). Thus, in view of the change of variables in equations (14), the limiting system (9) 
has no cycles in the region determined by

$$
-\varepsilon<X<\frac{\Lambda}{\mu}+\varepsilon \quad \text { and } \quad Y>-\varepsilon
$$

for some $\varepsilon>0$. We summarize these conclusions in the following theorem.

Theorem 2.1. Let $\left(x^{*}, y^{*}\right)$ denote the equilibrium point of the limiting system (15) that lies in the first quadrant, and whose coordinates satisfy the constraints in inequalities (21) and (22), and let $X^{*}$ and $Y^{*}$ be as given in equations (36). Let $R_{h}, R_{p}$, and $R_{0}$ denote the reproductive numbers defined in identities (11), (12) and (13), respectively. Assume that either (i) $R_{0}<1$, or (ii) $R_{0} \geqslant 1$ and $1-\phi<\frac{R_{h}+1}{R_{h}+R_{0}}$. Then, $\left(X^{*}, Y^{*}\right)$ is a locally, asymptotically stable equilibrium point of the limiting system (9). Furthermore, the system (9) has no cycles in the region defined by inequalities (37).

\section{Asymptotic Analysis of the Non-Autonomous System}

In this section we present an asymptotic analysis of the non-autonomous, twodimensional system (7). We have already noted in Section 1 that system (7) has a limiting system as $t \rightarrow \infty$ displayed in system (9). Thus, system (7) is an asymptotically autonomous system. Hence, the theory of asymptotically autonomous systems developed by Markus in [Mar56], and extended by Thieme in [Thi92] and [Thi94], and later by Castillo-Chavez and Thieme in [CCT95], can be applied to this situation. For the definitions of limiting systems and asymptotically autonomous systems, we refer the reader to [Mar56], [Thi92], [Thi94], and [CCT95]. It suffices to know for now that system (7) is an asymptotically autonomous system, and system (9) its limiting system.

First, note that in view of equation $(6), N_{p}(t)$ is bounded for all $t \geqslant t_{0}$. Furthermore, if we assume that

$$
0<N_{p_{0}}<\frac{\Lambda}{\mu}
$$

then it follows from equation (6) that

$$
0<N_{p}(t)<\frac{\Lambda}{\mu}, \quad \text { for all } t \geqslant t_{0} .
$$

Consider a solution of the non-autonomous system (7) subject to the initial condition

$$
\left(X_{p}\left(t_{0}\right), Y_{h}\left(t_{0}\right)\right)=\left(X_{0}, Y_{0}\right)
$$

for some $t_{0} \geqslant 0$, and $X_{0}$ and $Y_{0}$ satisfying

$$
0<X_{0}<\frac{\Lambda}{\mu} \text { and } 0<Y_{0}<N_{h} .
$$

We will also assume that the estimate on the initial patient population in inequality (38) is satisfied.

By the existence and uniqueness theorem for ordinary differential equations (see, for instance, [Hal09, Theorem 2.1, pg. 17]), there exists a unique solution of system (7),

$$
t \mapsto\left(X_{p}(t), Y_{h}(t)\right),
$$


satisfying the initial condition in equation (40), for $t$ in some maximal (forward) interval of existence $\left[t_{0}, T\right)$, for $T>t_{0}$. Denoting the solution in (42) by $\sigma(t)$, for $t \in\left[t_{0}, T\right)$, we will show that it will remain in the interior of the rectangular region

$$
\left[0, \frac{\Lambda}{\mu}\right] \times\left[0, N_{h}\right]
$$

for all $t \in\left[t_{0}, T\right)$, provided that the initial point $\left(X_{0}, Y_{0}\right)$ satisfies the estimates in inequalities (41), and the estimate in inequality (38) also holds true.

We first see that the trajectory $\sigma$ cannot leave the rectangle in (43) through the edge at $X_{p}=0$ (the $Y_{h}$-axis). Otherwise, there would be a time $t_{1} \in\left(t_{0}, T\right)$ such that

$$
X_{p}\left(t_{1}\right)=0 \quad \text { and } \quad X_{p}^{\prime}\left(t_{1}\right)<0 .
$$

However, according to the first differential equation in the non-autonomous system (7),

$$
X_{p}^{\prime}\left(t_{1}\right)=(1-\phi) \Lambda \geqslant 0,
$$

which contradicts the second statement in (44). We have therefore established that

$$
X_{p}(t)>0, \quad \text { for } t_{0} \leqslant t<T .
$$

In addition to the initial conditions in (38) and (39), we also assume that

$$
X_{h}\left(t_{0}\right)>0 \text { and } Y_{p}\left(t_{0}\right) \geqslant 0 .
$$

Next, we show that

$$
Y_{h}(t)>0, \quad \text { for } t_{0} \leqslant t<T,
$$

under the assumptions in (41).

If the claim in inequality (47) is not true, there exists $t_{1} \in\left(t_{0}, T\right)$ such that

$$
Y_{h}(t)>0, \quad \text { for } t \in\left[t_{0}, t_{1}\right) \text {, }
$$

and

$$
Y_{h}\left(t_{1}\right)=0 .
$$

It is also the case that

$$
Y_{h}^{\prime}\left(t_{1}\right)<0 .
$$

Now, it follows from the second equation in system (1), along with the statements (45) and (48), that

$$
\frac{d Y_{p}}{d t} \geqslant \phi \Lambda-\mu Y_{p}, \quad \text { for } t_{0} \leqslant t \leqslant t_{1},
$$

which we can rewrite as

$$
\frac{d Y_{p}}{d t}+\mu Y_{p} \geqslant \phi \Lambda, \quad \text { for } t_{0} \leqslant t \leqslant t_{1} .
$$

Multiplying the differential inequality (51) by $e^{\mu t}$, integrating from $t_{0}$ to $t$, and simplifying yields

$$
Y_{p}(t)>Y_{p}\left(t_{0}\right) e^{-\mu\left(t-t_{0}\right)}+\frac{\phi \Lambda}{\mu}\left(1-e^{-\mu\left(t-t_{0}\right)}\right), \quad \text { for } t_{0} \leqslant t \leqslant t_{1} .
$$

Consequently, in view of the second inequality in (46), we obtain from inequality (52) that

$$
Y_{p}(t)>0, \quad \text { for } t_{0} \leqslant t \leqslant t_{1} .
$$


Next, combine the statements (45) and (53) with the definition of $N_{p}(t)$ in equation (4) to deduce that

$$
X_{p}(t)<N_{p}(t), \quad \text { for } t_{0} \leqslant t \leqslant t_{1},
$$

from which we get that

$$
N_{p}(t)-X_{p}(t)>0, \quad \text { for } t_{0} \leqslant t \leqslant t_{1} .
$$

Substitute $t_{1}$ for $t$ in the second equation in system (7) to obtain that

$$
Y_{h}^{\prime}\left(t_{1}\right)=c b_{h}\left(N_{p}\left(t_{1}\right)-X_{p}\left(t_{1}\right)\right)\left(N_{h}-Y_{h}\left(t_{1}\right)\right)-\gamma Y_{h}\left(t_{1}\right) ;
$$

so that, using equation (49),

$$
Y_{h}^{\prime}\left(t_{1}\right)=c b_{h} N_{h}\left(N_{p}\left(t_{1}\right)-X_{p}\left(t_{1}\right)\right) .
$$

Consequently, by virtue of inequality (54), we see from the claim (55) that

$$
Y_{h}^{\prime}\left(t_{1}\right)>0 \text {, }
$$

which is in direct contradiction with the claim (50). We have therefore established the assertion in (47).

Note that in the previous argument we have also shown that

$$
Y_{p}(t)>0, \quad \text { for } t_{0} \leqslant t<T
$$

(see $(53))$.

It is also the case that

$$
X_{h}(t)>0, \quad \text { for } t_{0} \leqslant t<T,
$$

provided that the initial conditions in (46) are satisfied. For, if the assertion in (57) is not true, there exists $t_{1} \in\left(t_{0}, T\right)$ such that

$$
X_{h}\left(t_{1}\right)=0 .
$$

It is also the case that

$$
X_{h}^{\prime}\left(t_{1}\right)<0 .
$$

Consequently, substituting $t_{1}$ for $t$ in the third equation in system (1) and using equation (58), we obtain that

$$
X_{h}^{\prime}\left(t_{1}\right)=\gamma Y_{h}\left(t_{1}\right)
$$

so that, in view of the claim (47),

$$
X_{h}^{\prime}\left(t_{1}\right)>0,
$$

which is in direct contradiction with the claim (59). Hence, the assertion in (57) is established.

Now, it follows from the claims (45), (56) and the definition of $N_{p}(t)$ in equation (4) that

$$
0<X_{p}(t)<N_{p}(t), \quad \text { for } t_{0} \leqslant t<T ;
$$

so that, in view of inequality (39),

$$
0<X_{p}(t)<\frac{\Lambda}{\mu}, \quad \text { for } t_{0} \leqslant t<T .
$$

By the same token, in view of the claims (47), (57) and the definition of $N_{h}$ in equation (2),

$$
0<Y_{h}(t)<N_{h}, \quad \text { for } t_{0} \leqslant t<T \text {. }
$$


We have therefore established that any trajectory $\sigma$ of the non-autonomous system (7) that starts in the interior of the rectangle in (43) will remain in that rectangle for $t_{0} \leqslant t<T$, provided that the initial conditions in (46) also hold true.

Note that the vector field $F: \mathbb{R}^{2} \times[0, T) \rightarrow \mathbb{R}^{2}$ defined by

$F\left(X_{p}, Y_{h}, t\right)=\left(\begin{array}{c}(1-\phi) \Lambda-\mu X_{p}-c b_{p} Y_{h} X_{p} ; \\ c b_{h}\left(N_{p}(t)-X_{p}\right)\left(N_{h}-Y_{h}\right)-\gamma Y_{h}\end{array}\right), \quad$ for $\left(X_{p}, Y_{h}, t\right) \in \mathbb{R}^{2} \times[0, T)$, is $C^{\infty}$ and bounded over the set

$$
\left[0, \frac{\Lambda}{\mu}\right] \times\left[0, N_{h}\right] \times[0, T),
$$

in view of the fact that $N_{p}(t)$ is bounded for $t \geqslant t_{0}$ (see the definition of $N_{p}$ in equation (6)). Consequently, since every trajectory, $\sigma(t)$, for $t \in\left[t_{0}, T\right)$, of system (7), where $\left[t_{0}, T\right)$ is the maximal (forward) interval of existence, that starts in the interior of the rectangle in (43) remains in that rectangle for all $t \in\left[t_{0}, T\right)$, it must be the case that $T=\infty$. Otherwise we would be able to extend the trajectory past $t=T<\infty$, contradicting the maximality of the interval $\left[t_{0}, T\right.$ ) (see, for instance, Corollary 3.2 on page 14 in [Har82]). Hence, every trajectory, $\sigma(t)$, of the nonautonomous system (7) that starts in the interior of the rectangle in (43) exists for all $t \geqslant t_{0}$ and remains in that rectangle for all $t \geqslant t_{0}$. We can therefore discuss the $\omega$-limit set of $\sigma$.

Consider a general two-dimensional system of differential equations

$$
\left\{\begin{array}{l}
\dot{x}_{1}=f_{1}\left(x_{1}, x_{2}, t\right) \\
\dot{x}_{2}=f_{2}\left(x_{1}, x_{2}, t\right)
\end{array}\right.
$$

with $f_{1}$ and $f_{2}$ being continuous real-valued functions defined on $R \times\left[t_{0}, \infty\right)$, where $R$ is a region in $\mathbb{R}^{2}$ and $t_{0}$ is some real number. Given $\left(x_{1_{0}}, x_{2_{0}}\right) \in R$, suppose that system (62) has a solution, $\sigma(t)=\left(x_{1}(t), x_{2}(t)\right)$, satisfying

$$
x_{1}\left(t_{0}\right)=x_{1_{0}} \quad \text { and } \quad x_{2}\left(t_{0}\right)=x_{2_{0}},
$$

that is unique and exists for all $t \geqslant t_{0}$. Suppose further that the functions $f_{1}$ and $f_{2}$ in system (62) are locally Lipschitz continuous with respect to the variables $x_{1}$ and $x_{2}$. For the case in which the trajectory $\sigma(t)$, for $t \geqslant t_{0}$, is also assumed to be bounded, the $\omega$-limit set of $\sigma$, denoted $\omega(\sigma)$, is defined as follows:

$$
\left(y_{1}, y_{2}\right) \in \omega(\sigma) \Leftrightarrow y_{1}=\lim _{j \rightarrow \infty} x_{1}\left(t_{j}\right) \text { and } y_{2}=\lim _{j \rightarrow \infty} x_{2}\left(t_{j}\right)
$$

for some sequence $\left(t_{j}\right)$ of real numbers in $\left[t_{0}, \infty\right)$ such that $t_{j} \rightarrow \infty$ as $j \rightarrow \infty$.

Markus [Mar56] proved that, if $\sigma(t)$ for $t \geqslant t_{0}$ is bounded, then the $\omega$-limit set $\omega(\sigma)$ is nonempty, compact, and connected. It is possible to characterize the $\omega$-limit set of a bounded trajectory of the system (62) for the case in which the system (62) is asymptotically autonomous, as shown in [Mar56], [Thi92], [Thi94], and [CCT95].

The system (62) is said to be asymptotically autonomous if there exists an autonomous system

$$
\left\{\begin{array}{l}
\dot{y}_{1}=g_{1}\left(y_{1}, y_{2}\right) \\
\dot{y}_{2}=g_{2}\left(y_{1}, y_{2}\right),
\end{array}\right.
$$

where $g_{1}$ and $g_{2}$ are real-valued functions that are locally Lipschitz continuous in some plane region containing $R$, such that

$$
f_{1}\left(x_{1}, x_{2}, t\right) \rightarrow g_{1}\left(x_{1}, x_{2}\right) \quad \text { and } \quad f_{2}\left(x_{1}, x_{2}, t\right) \rightarrow g_{2}\left(x_{1}, x_{2}\right), \quad \text { as } t \rightarrow \infty
$$


locally uniformly in $\left(x_{1}, x_{2}\right) \in R$.

For the situation described above in the asymptotically autonomous system (62) with limiting system (63), it is reasonable to expect that the structure of the $\omega$-limit sets of bounded (forward) orbits for the non-autonomous system (62) is dictated by the structure of the $\omega$-limit sets of the limiting system (63). This is indeed the case. In fact, there is a Poincaré-Bendixson type trichotomy result established by Thieme in [Thi92] and [Thi94]. We quote here the version of such result found in [CCT95, Theorem 2.1] in the context of the asymptotically autonomous system (62) and its limiting system (63).

Theorem 3.1 (Thieme, 1992). Let $\sigma$ be a forward bounded orbit of the asymptotically autonomous system (62). Let $\omega(\sigma)$ denote its $\omega$-limit set and assume that there exists a neighborhood of $\omega(\sigma)$ that contains at most finitely many equilibrium points of the limiting system (63). Then, one of the following holds true:

(i) $\omega(\sigma)$ consists of an equilibrium point of system (63).

(ii) $\omega(\sigma)$ is the union of periodic orbits of system (63) and possibly of centers of system (63) that are surrounded by periodic orbits of system (63) lying in $\omega(\sigma)$.

(iii) $\omega(\sigma)$ contains equilibrium points of system (63) that are cyclically chained to each other in $\omega(\sigma)$ by orbits of system (63).

In this section we will prove that, under the assumptions of Theorem 2.1 in Section 2 of this paper, every bounded forward trajectory, $\sigma(t)$, of the non-autonomous system (7) that starts in the interior of the rectangle in (43) converges to the locally asymptotically stable equilibrium point, $\left(X^{*}, Y^{*}\right)$, of the limiting system (9) given in Theorem 2.1. We will do this by precluding options (ii) and (iii) in the trichotomy laid out by Theorem 3.1. To accomplish this, we follow the argument outlined in the corollary to Theorem 3.1 given in [CCT95, Corollary 2.2].

Corollary 3.2 (Corollary 2.2 in [CCT95]). For the asymptotically autonomous system (62) with limiting system (63), assume that there is subset $R$ of $\mathbb{R}^{2}$ such that any equilibrium point of system (63) in $R$ is the only equilibrium point in a sufficiently small neighborhood. Furthermore, assume that there exist a subset $S$ of $\mathbb{R}^{2}$ and an open simply connected subset $\Omega$ of $\mathbb{R}^{2}$ with the following properties:

- Every forward bounded orbit of system (62) in $R$ has its $\omega$-limit set in $S$.

- All possible periodic orbits of system (63) in $S$ and the closures of all possible orbits of system (63) that chain equilibrium points of system (63) cyclically in $S$ are contained in $\Omega$.

- The vector field $G=\left(g_{1}, g_{2}\right)$ is $C^{1}$ in $\Omega$ and there is a real-valued $C^{1}$ function $\rho$ in $\Omega$ such that $\operatorname{div}(\rho G)$ is either strictly positive almost everywhere on $\Omega$ or strictly negative almost everywhere in $\Omega$.

Then, every forward bounded solution of system (62) in $R$ and every forward bounded solution of system (63) in $R$ converges towards an equilibrium point of system (63) as $t \rightarrow \infty$.

We are now in a position to state and prove the main result of this section.

Theorem 3.3. Let $\left(X^{*}, Y^{*}\right)$ be as defined in equations (36), where $\left(x^{*}, y^{*}\right)$ denotes the equilibrium point of the system (15) that lies in the first quadrant, and whose coordinates satisfy the constraints in (21) and (22). Let $R_{h}, R_{p}$, and $R_{0}$ denote the reproductive numbers defined in equations (11), (12) and (13), respectively. Assume 
that either ( $i$ ) $R_{0}<1$, or (ii) $R_{0} \geqslant 1$ and $1-\phi<\frac{R_{h}+1}{R_{h}+R_{0}}$. Then, any trajectory $\sigma(t)$ of the non-autonomous system ( 7$)$ that starts in the interior of the rectangle in (43) will converge toward the equilibrium point $\left(X^{*}, Y^{*}\right)$ as $t \rightarrow \infty$.

Proof: Let $S$ denote the rectangle in (43) and $R$ denote the interior of $S$. Define $\Omega$ to be the set

$$
\Omega=\left\{(X, Y) \in \mathbb{R}^{2} \mid-\varepsilon<X<\frac{\Lambda}{\mu}+\varepsilon \quad \text { and } \quad Y>-\varepsilon\right\},
$$

where $\varepsilon>0$ is as given in Theorem 2.1. We may assume that $\varepsilon$ is sufficiently small so that $\Omega$ contains only the equilibrium point $\left(X^{*}, Y^{*}\right)$ of the limiting system in (9) given by Theorem 2.1. Note that $\Omega$ is open and simply connected.

We have shown in this section that any trajectory $\sigma(t)$ of the non-autonomous system (7) that starts in $R$ at time $t_{0}$ will remain in $R$ for all $t \geqslant t_{0}$, provided that the initial conditions in (46) also hold true. Consequently, the $\omega$-limit set of $\sigma$ will be in $S$. Thus, the first bullet point in Corollary 3.2 holds true.

We have already pointed out in Theorem 2.1 that the limiting system (9) has no cycles in $S$. Furthermore, since $\Omega$ contains only one equilibrium point of the limiting system (9) (namely, the point $\left(X^{*}, Y^{*}\right)$ ), there are no cyclically chained equilibrium points of system (9). Thus, the condition in the second bullet point in Corollary 3.2 is vacuously satisfied.

The third bullet point condition in Corollary 3.2 on the divergence of $\rho G$ has already been verified in the arguments leading to the proof of Theorem 2.1 for the case $\rho \equiv 1$; see the statement in $(35)$.

Hence, Corollary 3.2 can be applied to the asymptotically autonomous system (7) and its limiting system (9) to conclude that any trajectory $\sigma(t)$ of the nonautonomous system (7) that starts in $R$ will converge toward the equilibrium point $\left(X^{*}, Y^{*}\right)$ as $t \rightarrow \infty$.

\section{Analysis of the Austin-Anderson AmR Transmission Model}

In this section we return to the Austin-Anderson AMR transmission model (1) that we introduced in Section 1 and analyzed its associated systems in Sections 2 and 3. While in Section 3 we analyzed the asymptotically autonomous system (7) involving the variables $X_{p}$ and $Y_{h}$, we can achieve a result similar to that in [AA99] by analyzing the asymptotically autonomous system involving $Y_{p}$ and $Y_{h}$. Consider the following system

$$
\left\{\begin{aligned}
\frac{d Y_{p}}{d t} & =\phi \Lambda-\mu Y_{p}+c b_{p} Y_{h}\left(N_{p}(t)-Y_{p}\right) \\
\frac{d Y_{h}}{d t} & =c b_{h} Y_{p}\left(N_{h}-Y_{h}\right)-\gamma Y_{h}
\end{aligned}\right.
$$

where $N_{p}(t)$ is given in equation (6) and $N_{h}$ is the constant number of HCWs.

By virtue of equation (8), the non-autonomous system (64) has a limiting system

$$
\left\{\begin{aligned}
\frac{d W}{d t} & =\phi \Lambda-\mu W+c b_{p} Z\left(\frac{\Lambda}{\mu}-W\right) ; \\
\frac{d Z}{d t} & =c b_{h} W\left(N_{h}-Z\right)-\gamma Z
\end{aligned}\right.
$$


as $t \rightarrow \infty$, which is an autonomous system. Note that in this case, the variable $W$ in limiting system (65) corresponds to the number of colonized patients, while $Z$ corresponds to the number of colonized HCWs.

Using the dimensionless variables in equations (14), we can rewrite the system (65) in dimensionless form

$$
\left\{\begin{aligned}
\frac{d w}{d \tau} & =\phi-w+\alpha_{1}(1-w) z \\
\frac{d z}{d \tau} & =\alpha_{2} w(1-z)-\alpha_{3} z
\end{aligned}\right.
$$

where

$$
\alpha_{1}=\frac{c b_{p} N_{h}}{\mu}, \quad \alpha_{2}=\frac{c b_{h} \bar{N}_{p}}{\mu}, \quad \text { and } \quad \alpha_{3}=\frac{\gamma}{\mu}
$$

are the same dimensionless parameters defined in equations (16), (17), and (18), respectively.

Figure 4 shows a sketch of the nullclines of system (66). The $w$-nullcline of

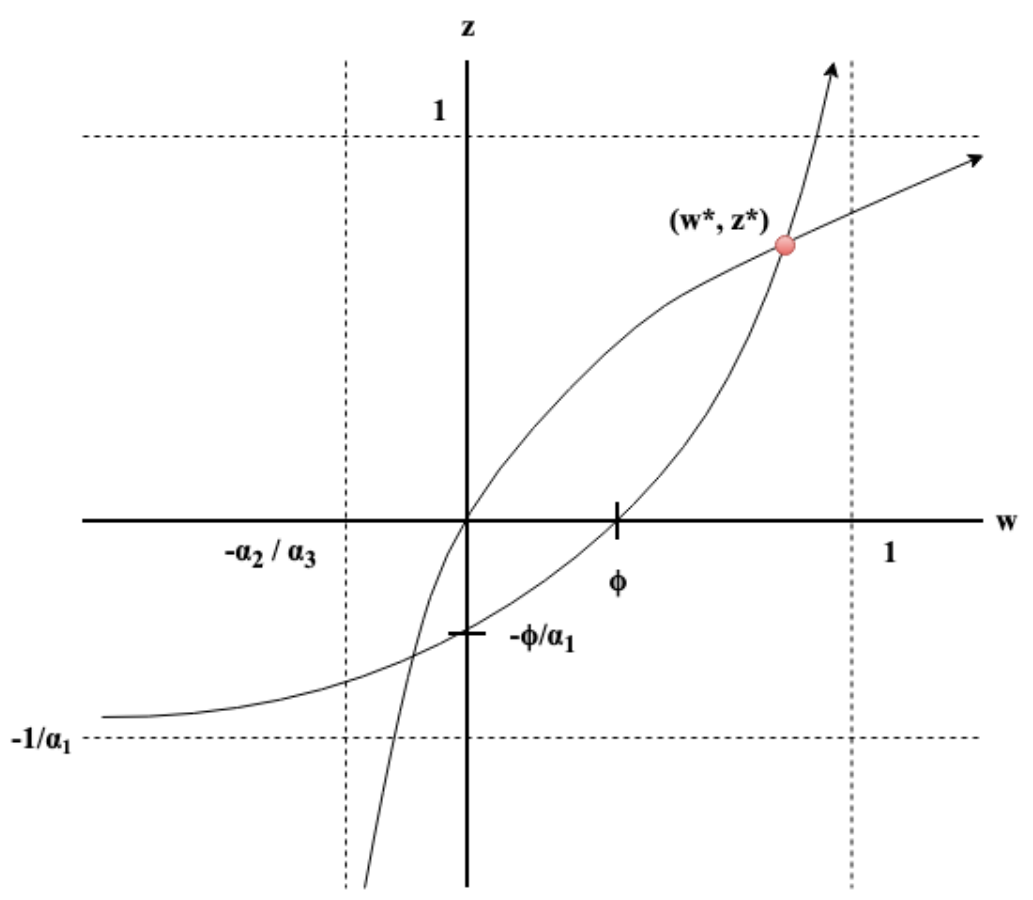

Figure 4. Nullclines of the limiting system (66).

system (66) is the graph of the equation

$$
z=\frac{w-\phi}{\alpha_{1}(1-w)}
$$


which has the line $w=1$ as a vertical asymptote, and the line $z=-\frac{1}{\alpha_{1}}$ as a horizontal asymptote (shown as a dotted lines in Figure 4). Observe that this curve has $\phi$ as its $w$-intercept.

The $z$-nullcline of system (66) is the graph of the equation

$$
z=\frac{\alpha_{2} w}{\alpha_{3}+\alpha_{2} w}
$$

which has the line $z=1$ as a horizontal asymptote, and the line $w=-\frac{\alpha_{3}}{\alpha_{2}}$ as a vertical asymptote (these are shown as dotted lines in Figure 4). Note that the $z$-nullcline has the origin $(w=0$ and $z=0)$ as the $w$-intercept and $z$-intercept.

The analysis of the asymptotically autonomous system (64) based on the analysis of its limiting system (65) presented in this section and the Appendix will serve as another example of the application of the theory of asymptotically autonomous systems discussed in Sections 2 and 3. Since the analysis of the limiting system (65) closely parallels that of the limiting system (9), we leave the remaining details in the Appendix. The local stability result for the limiting system (65) is as follows:

Theorem 4.1. Let $R_{h}, R_{p}$, and $R_{0}$ denote the reproductive numbers defined in identities (11), (12), and (13), respectively. Assume that either (i) $R_{0}<1$, or (ii) $R_{0} \geqslant 1$ and $\phi>\frac{R_{0}-1}{R_{h}+R_{0}}$. Then system (65) has an equilibrium point $\left(Y_{p}^{*}, Y_{h}^{*}\right)$ satisfying

$$
\frac{\Lambda \phi}{\mu}<Y_{p}^{*}<\frac{\Lambda}{\mu} \quad \text { and } \quad 0<Y_{h}^{*}<N_{h}
$$

which is locally, asymptotically stable. Furthermore, system (65) has no cycles in the region defined by

$$
-\varepsilon<W<\frac{\Lambda}{\mu}+\varepsilon \quad \text { and } \quad-\varepsilon<Z<N_{h}+\varepsilon,
$$

for some $\varepsilon>0$.

Next, we may proceed as Section 3 in this paper to deduce the following local stability result for the asymptotically autonomous system (64):

Theorem 4.2. Let $R_{h}, R_{p}$, and $R_{0}$ denote the reproductive numbers defined in identities (11), (12), and (13), respectively, and assume that either (i) $R_{0}<1$, or (ii) $R_{0} \geqslant 1$ and $\phi>\frac{R_{0}-1}{R_{h}+R_{0}}$. Let $\left(Y_{p}^{*}, Y_{h}^{*}\right)$ be the equilibrium point of system (65) given by Theorem 4.1. Then any trajectory $\sigma(t)$ of the non-autonomous system (64) that starts in the interior of the rectangle in (43) will converge toward the equilibrium point $\left(Y_{p}^{*}, Y_{h}^{*}\right)$ as $t \rightarrow \infty$.

Remark 4.3. The proof of Theorem 4.2 can be achieved by verifying the conditions in Corollary 3.2, which was expounded in Section 3 in the context of the theory of asymptotically autonomous systems developed by Markus in [Mar56], and extended by Thieme in [Thi92] and [Thi94], and later by Castillo-Chavez and Thieme in [CCT95]. 
We can compute the coordinates of the equilibrium point $\left(Y_{p}^{*}, Y_{h}^{*}\right)$ given by Theorem 4.2 by first computing the coordinates of the equilibrium point $\left(w^{*}, z^{*}\right)$ of system (66) in the first quadrant, and then using the expressions

$$
Y_{p}^{*}=\frac{\Lambda}{\mu} w^{*} \quad \text { and } \quad Y_{h}^{*}=N_{h} z^{*} .
$$

To compute $\left(w^{*}, z^{*}\right)$, the intersection in the first quadrant of the nullclines of the system in (66), we solve the system of equations in (67) and (68) simultaneously, and select the solution in the first quadrant. For instance, setting the expressions for $z$ in equations (67) and (68) equal to each other leads to the quadratic equation

$$
\alpha_{2}\left(\alpha_{1}+1\right) w^{2}+\left(\alpha_{3}-\alpha_{2}\left(\alpha_{1}+\phi\right)\right) w-\alpha_{3} \phi=0 .
$$

The positive solution of the equation (70) is

$$
w^{*}=\frac{\alpha_{2}\left(\alpha_{1}+\phi\right)-\alpha_{3}+\sqrt{\left(\alpha_{2}\left(\alpha_{1}+\phi\right)-\alpha_{3}\right)^{2}+4 \alpha_{2} \alpha_{3}\left(\alpha_{1}+1\right)}}{2 \alpha_{2}\left(\alpha_{1}+1\right)},
$$

which we can rewrite in terms of the reproductive numbers $R_{h}, R_{p}$, and $R_{0}$ defined in equations (11), (12), and (13), respectively, as

$$
w^{*}=\frac{R_{0}-1+R_{h} \phi+\sqrt{\left(R_{0}-1+R_{h} \phi\right)^{2}+4 R_{h}\left(1+R_{p}\right) \phi}}{2 R_{h}\left(1+R_{p}\right)},
$$

or

$$
w^{*}=\frac{R_{0}-1+R_{h} \phi+\sqrt{\left(R_{0}-1+R_{h} \phi\right)^{2}+4\left(R_{h}+R_{0}\right) \phi}}{2\left(R_{h}+R_{0}\right)},
$$

where we have used the identities in (19) and (20). We note that the expression in (71) is equation (31) in [AA99] for the prevalence of colonizations in patients in the hospital unit.

The second coordinate of the equilibrium point of system (66) that is in the first quadrant is then given by

$$
z^{*}=\frac{w^{*}-\phi}{R_{p}\left(1-w^{*}\right)},
$$

according to equations (67) and (19), where $w^{*}$ is given in equation (71).

Using the expressions in (69) we get values for $Y_{p}^{*}$ and $Y_{h}^{*}$, where $w^{*}$ is given in equation (71), and $z^{*}$ in equation (72).

Next, use the definitions of $N_{p}(t)$ and $N_{h}$ in equations (4) and (2), respectively, and the limit fact in (8) to conclude that

$$
X_{p}^{*}=\frac{\Lambda}{\mu}-Y_{p}^{*} \quad \text { and } \quad X_{h}^{*}=N_{h}-Y_{h}^{*} .
$$

Note that $\left(X_{p}^{*}, Y_{h}^{*}\right)$ is the asymptotically stable equilibrium point system (7), which was analyzed in Section 3, provided that the conditions in Theorem 3.3 hold true.

Therefore, the expressions in (69) and (73) yield the coordinates, $\left(X_{p}^{*}, Y_{p}^{*}, X_{h}^{*}, Y_{h}^{*}\right)$, of an equilibrium point of the Austin-Anderson system (1) that lies in the interior of the region

$$
\left[0, \frac{\Lambda}{\mu}\right] \times\left[0, \frac{\Lambda}{\mu}\right] \times\left[0, N_{h}\right] \times\left[0, N_{h}\right]
$$


in four-dimensional Euclidean space. If the hypotheses of Theorem 4.2 (or Theorem 4.1) hold true, then the equilibrium point

$$
\left(\frac{\Lambda}{\mu}-Y_{p}^{*}, Y_{p}^{*}, N_{h}-Y_{h}^{*}, Y_{h}^{*}\right)
$$

is asymptotically stable. Furthermore, any trajectory, $\left(X_{p}(t), Y_{p}(t), X_{h}(t), Y_{h}(t)\right)$, for $t \geqslant 0$, of the Austin-Anderson system (1) that starts at a point $\left(X_{p}(0), Y_{p}(0), X_{h}(0), Y_{h}(0)\right)$ in the interior of the region in (74) will tend towards the equilibrium point in (75) as $t \rightarrow \infty$.

\section{Conclusion}

This paper presents an analysis of a four-dimensional compartmental model of AMR transmission introduced by Austin and Anderson [AA99]. In particular, our analysis provides a mathematical justification for Austin and Anderson's stability analysis. In the Austin-Anderson model, patients and HCWs act as hosts and vectors of AMR microorganisms, respectively; in turn, these are subdivided into uncolonized and colonized groups. To better understand the model, we reduce the system's dimension and arrive at a two-dimensional yet non-autonomous system. Furthermore, this system has an autonomous limiting system, for which we prove the existence of a locally asymptotically stable equilibrium point under a set of constraints expressed in terms of reproductive numbers.

The stability analysis of the Austin-Anderson system presented in [AA99] was established on a limiting system. Here comes a challenging aspect of the analysis, however, because it is not immediately apparent to determine the stability properties of the non-autonomous system based on the properties of autonomous limiting system. The workaround is to apply the theory of asymptotically autonomous differential equations from [Mar56], [Thi92], [Thi94] and a Poincaré-Bendixson type trichotomy result from [CCT95] to the non-autonomous system. The outcomes are Theorems 4.1 and 4.2, of which their hypotheses help us establish two facts:

- the equilibrium point of the Austin-Anderson system is locally asymptotically stable;

- any forward bounded trajectory of the non-autonomous system that starts in the interior of a defined rectangular region will tend towards the equilibrium point of the limiting system.

Within the scope of the Austin-Anderson model, these findings tell us that we can define the threshold of AMR transmission in an ICU environment by generating conditions for which an endemic equilibrium exists and is locally asymptotically stable. The conditions involve model-specific parameters such as reproductive numbers, patient admission rate, prevalence of colonization at admission, and patient's average length of stay. In order for hospital management and researchers to achieve an effective disease surveillance, it would be crucial to reliably measure these statistics and interpret the conditions for an endemic equilibrium to exist. Future extensions of this work may include bringing epidemiological insights to better interpret the parameter-based conditions. 


\section{REFERENCES}

[AA99] D. J. Austin and R. M. Anderson. Studies of antibiotic resistance within the patient, hospitals and the community using simple mathematical models. Phil. Trans. of the $R$. Soc. of Lond. B: Biol. Sci., 354(1384):721-738, 1999.

[Arn92] V.I. Arnold. Ordinary Differential Equations. Springer Textbook. Springer Berlin Heidelberg, 1992.

[BW15] C. Browne and G.F. Webb. A nosocomial epidemic model with infection of patients due to contaminated rooms. Math. Bio. and Engin., 12(4):761-787, 2015.

[CCT95] C. Castillo-Chavez and H. R. Thieme. Asymptotically autonomous epidemic models. In O. Arino, D. Axelrod, M. Kimmel, and M. Langlais, editors, Mathematical Population Dynamics: Analysis of Heterogeneity, Volume 1, Theory of Epidemics, pages 33-50. Wuerz Publishers, 1995.

[EDW07] D. Olivier S. Ruan E.M.C. D'Agata, P. Magal and G. Webb. Modeling antibiotic resistance in hospitals: The impact of minimizing treatment duration. J. The. Biol., 249(3):487-499, 2007.

[Hal09] J. K. Hale. Ordinary Differential Equations. Dover Publications, Dover edition, 2009.

[Har82] P. Hartman. Ordinary Differential Equations. Birkäuser, second edition, 1982.

[Mar56] L. Markus. Asymptotically autonomous differential systems. In S. Lefschetz, editor, Contributions to the Theory of Nonlinear Oscillations (AM-36), Volume III, pages 17-30. Princeton University Press, 1956.

[MD13] Krisztian Magori and John M. Drake. The population dynamics of vector-borne diseases. Nature Educ., 2013.

[Str98] Marc J Struelens. The epidemiology of antimicrobial resistance in hospital acquired infections: problems and possible solutions, chapter 317.7159, pages 652-654. BMJ: British Medical Journal, 1998.

[Str00] S. H. Strogatz. Nonlinear Dynamics And Chaos: With Applications To Physics, Biology, Chemistry, And Engineering. Studies in Nonlinearity. Westview Press, 1st edition, 2000.

[Thi92] H. R. Thieme. Convergence results and a poincar-bendixson trichotomy for asymptotically autonomous differential equations. J. Math. Biol., 30:755-763, 1992.

[Thi94] H. R. Thieme. Asymptotically autonomous differential equations in the plane. ii. stricter poincar/bendixson type results. Diff. and Int. Eq., 7(5-6):1625-1640, 1994.

\section{APPENDIX}

The analysis of the asymptotically autonomous system (64) based on the analysis of its limiting system (65) presented in this section will serve as another example of the application of the theory of asymptotically autonomous systems discussed in Section (3).

The sketch in Figure 4 shows that the $w$-nullcline and the $z$-nullcline intersect at a point below the $w$-axis in the third quadrant and at a point in the first quadrant that we denote by $\left(w^{*}, z^{*}\right)$. There are other branches of the nullclines which do not intersect and therefore are not shown in Figure 4. Thus, the limiting system (66) has two equilibrium points. We will focus our attention on the equilibrium point $\left(w^{*}, z^{*}\right)$ in the first quadrant. We first note that the coordinates of this equilibrium point satisfy the estimates

$$
0<z^{*}<1
$$

and

$$
\phi<w^{*}<1
$$

We will next determine parameter conditions that will guarantee that the equilibrium point $\left(w^{*}, z^{*}\right)$ is (locally) asymptotically stable. To do this we apply the 
Principle of Linearized Stability to the vector field

$$
F(w, z)=\left(\begin{array}{c}
\phi-w+\alpha_{1}(1-w) z \\
\alpha_{2} w(1-z)-\alpha_{3} z
\end{array}\right), \quad \text { for }(w, z) \in \mathbb{R}^{2},
$$

at the equilibrium point $\left(w^{*}, z^{*}\right)$.

The derivative of the map $F: \mathbb{R}^{2} \rightarrow \mathbb{R}^{2}$ defined in the vector field (78) evaluated at $\left(w^{*}, z^{*}\right)$ is

$$
D F\left(w^{*}, z^{*}\right)=\left(\begin{array}{cc}
-1-\alpha_{1} z^{*} & \alpha_{1}\left(1-w^{*}\right) \\
\alpha_{2}\left(1-z^{*}\right) & -\alpha_{2} w^{*}-\alpha_{3}
\end{array}\right) .
$$

The trace of the Jacobian matrix in (79) is

$$
\tau\left(w^{*}, z^{*}\right)=-1-\alpha_{1} z^{*}-\alpha_{2} w^{*}-\alpha_{3}
$$

and the determinant is

$$
\Delta\left(w^{*}, z^{*}\right)=\left(1+\alpha_{1} z^{*}\right)\left(\alpha_{2} w^{*}+\alpha_{3}\right)-\alpha_{1} \alpha_{2}\left(1-w^{*}\right)\left(1-z^{*}\right) .
$$

From the constraints on the coordinates of $\left(w^{*}, z^{*}\right)$ in inequalities (76) and (77), and the assumption that the parameters $\alpha_{1}, \alpha_{2}$, and $\alpha_{3}$ are positive, it follows from equation (80) that

$$
\tau\left(w^{*}, z^{*}\right)<0 .
$$

Next, we use the fact that $0<z^{*}<1$, according to inequality (76), to estimate

$$
\frac{\Delta\left(w^{*}, z^{*}\right)}{1-z^{*}}=\frac{1+\alpha_{1} z^{*}}{1-z^{*}}\left(\alpha_{2} w^{*}+\alpha_{3}\right)-\alpha_{1} \alpha_{2}\left(1-w^{*}\right) .
$$

Using the fact that

$$
\frac{1+\alpha_{1} z}{1-z} \geqslant 1, \quad \text { for } 0 \leqslant z<1
$$

we get

$$
\frac{\Delta\left(w^{*}, z^{*}\right)}{1-z^{*}} \geqslant \alpha_{2} w^{*}+\alpha_{3}-\alpha_{1} \alpha_{2}\left(1-w^{*}\right),
$$

or

$$
\frac{\Delta\left(w^{*}, z^{*}\right)}{1-z^{*}} \geqslant\left(\alpha_{2}+\alpha_{1} \alpha_{2}\right) w^{*}+\alpha_{3}-\alpha_{1} \alpha_{2}
$$

Observe that

$$
z=\left(\alpha_{2}+\alpha_{1} \alpha_{2}\right) w+\alpha_{3}-\alpha_{1} \alpha_{2}
$$

is the equation of a straight line with $z$-intercept $\alpha_{3}-\alpha_{1} \alpha_{2}$ and $w$-intercept

$$
\frac{\alpha_{1} \alpha_{2}-\alpha_{3}}{\alpha_{2}\left(1+\alpha_{1}\right)}=\frac{\alpha_{1}-\frac{\alpha_{3}}{\alpha_{2}}}{1+\alpha_{1}}=\frac{R_{p}-\frac{1}{R_{h}}}{1+R_{p}}=\frac{R_{0}-1}{R_{h}+R_{0}},
$$

where we have used the identities in (19) and (20).

We consider two possibilities:

(i) $\alpha_{3}-\alpha_{1} \alpha_{2}>0$, or

(ii) $\alpha_{3}-\alpha_{1} \alpha_{2} \leqslant 0$.

Cases (i) and (ii) above are equivalent to the cases

(i) $R_{0}<1$, or

(ii) $R_{0} \geqslant 1$, 
where $R_{0}$ is the reproductive number defined in equation (13), in view of the identities in (19) and (20).

Note that the straight line in equation (84) has positive slope. Thus, in case (i) we have that

$$
\frac{\Delta\left(w^{*}, z^{*}\right)}{1-z^{*}}>0, \quad \text { provided that } w^{*}>0 \quad \text { and } \quad 0<z^{*}<1,
$$

regardless of the value of $\phi$. Consequently,

$$
\Delta\left(w^{*}, z^{*}\right)>0, \quad \text { provided that } R_{0}<1,
$$

in view of the fact that the coordinates of the equilibrium point $\left(w^{*}, z^{*}\right)$ satisfy the estimates in inequalities (76) and (77).

On the other hand, in case (ii) above, it follows from inequality (83) that

$$
\frac{\Delta\left(w^{*}, z^{*}\right)}{1-z^{*}}>0, \quad \text { provided that } w^{*}>\frac{R_{0}-1}{R_{h}+R_{0}},
$$

since the expression on the right-hand side of inequality (83) is positive. Since the straight line (84) has positive slope, such observation can be made provided that $w^{*}$ lies to the right-hand side of the $w$-intercept of the line (84).

Thus, by virtue of the estimate for $w^{*}$ in inequality (77), we obtain from the claim (87) that

$$
\frac{\Delta\left(w^{*}, z^{*}\right)}{1-z^{*}}>0, \quad \text { provided that } \phi>\frac{R_{0}-1}{R_{h}+R_{0}},
$$

from which we obtain that

$$
\Delta\left(w^{*}, z^{*}\right)>0, \quad \text { provided that } \phi>\frac{R_{0}-1}{R_{h}+R_{0}},
$$

given the estimate for $z^{*}$ in inequality (76). It then follows from the claim (88) that

$$
\Delta\left(w^{*}, z^{*}\right)>0, \quad \text { provided that } R_{0} \geqslant 1 \text { and } \phi>\frac{R_{0}-1}{R_{h}+R_{0}} .
$$

Combining claims (86) and (89) together, we conclude that the determinant of the linearization (79) of the limiting system (66) is positive in the cases

$$
\begin{aligned}
& \text { (i) } R_{0}<1 \text {, or } \\
& \text { (ii) } R_{0} \geqslant 1 \text { and } \phi>\frac{R_{0}-1}{R_{h}+R_{0}} \text {. }
\end{aligned}
$$

Since the trace of the matrix in (79) is negative, according to inequality (82), the real parts of the eigenvalues of the linearization of the limiting system (66) are negative in the cases (90) or (91) given above. Consequently, by the Principle of Linearized Stability, the equilibrium point $\left(w^{*}, z^{*}\right)$ is (locally) asymptotically stable. Thus, as in the arguments leading to the proof of Theorem 2.1 in Section 2 of this paper, we have arrived at a local stability result for limiting system (65).

Remark 5.1. The last assertion in the statement of Theorem 4.1 follows from the Bendixson-Dulac Criterion and the fact that the divergence of the vector field $F$ given in (78),

satisfies

$$
\operatorname{div} F(w, z)=-1-\alpha_{1} z-\alpha_{2} w-\alpha_{3}
$$

$$
\operatorname{div} F(w, z) \leqslant-1 \text { for } w \geqslant 0 \text { and } z \geqslant 0 .
$$


Remark 5.2. We note that the conditions in (90) and (91) are the same as the conditions in Theorem 2.1 in Section 2 of this paper, since

$$
\phi>\frac{R_{0}-1}{R_{h}+R_{0}}
$$

is equivalent to

$$
1-\phi<\frac{R_{h}+1}{R_{h}+R_{0}} .
$$

Thus, Theorem 4.1 can be obtained as a consequence of Theorem 2.1 by noting that the equilibrium point $\left(Y_{p}^{*}, Y_{h}^{*}\right)$ given in Theorem 4.1 is given by

$$
Y_{p}^{*}=\frac{\Lambda}{\mu}-W^{*} \quad \text { and } \quad Y_{h}^{*}=Z^{*},
$$

where $\left(W^{*}, Z^{*}\right)$ is the equilibrium point given by Theorem 2.1. This assertion is justified by the definition of $N_{p}(t)$ in equation (4) and its asymptotic expression in (8). 